\title{
Long-term outcomes of a totally thoracoscopic approach for reoperative mitral valve replacement: a propensity score matched analysis
}

\author{
Peijian Wei ${ }^{1,2 \#}$, Jian Liu ${ }^{1 \#}$, Jiexu Ma ${ }^{1,2 \#}$, Yuyuan Zhang ${ }^{1}$, Zhao Chen ${ }^{1}$, Yanjun Liu ${ }^{1}$, Tong Tan ${ }^{1,2}$, \\ Hongxiang $\mathrm{Wu}^{1}$, Jimei Chen ${ }^{1}$, Jian Zhuang ${ }^{1}$, Huiming Guo ${ }^{1}$
}

${ }^{1}$ Department of Cardiovascular Surgery, Guangdong Provincial Key Laboratory of South China Structural Heart Disease, Guangdong Cardiovascular Institute, Guangdong Provincial People's Hospital (Guangdong Academy of Medical Sciences), Guangzhou, China; ${ }^{2}$ Shantou University Medical College, Shantou, China

Contributions: (I) Conception and design: P Wei, J Liu, J Ma; (II) Administrative support: J Liu, J Chen, J Zhuang, H Guo; (III) Provision of study materials or patients: Y Zhang, Z Chen; (IV) Collection and assembly of data: Y Liu, T Tan, H Wu; (V) Data analysis and interpretation: P Wei, J Liu; (VI) Manuscript writing: All authors; (VII) Final approval of manuscript: All authors.

\#These authors contributed equally to this work.

Correspondence to: Huiming Guo; Jian Liu. Department of Cardiovascular Surgery, Guangdong Provincial Key Laboratory of South China Structural Heart Disease, Guangdong Cardiovascular Institute, Guangdong Provincial People's Hospital (Guangdong Academy of Medical Sciences), Guangzhou 510080, China. Email: guohuiming@gdph.org.cn; Jameslau1984@sina.com.

Background: This study aimed to summarize the perioperative and long-term outcomes of patients with previous mitral valve surgery (MVS) undergoing reoperative mitral valve replacement (MVR).

Methods: Data for all reoperative mitral valve replacements (re-MVRs) with or without concomitant tricuspid surgery were analyzed from Guangdong Provincial People's Hospital between January 2013 and December 2019. Propensity score matching resulted in 30 matched pairs with improved balance after matching in baseline covariates. Perioperative data and long term clinical outcomes were analyzed.

Results: Results are based on the matched cohorts between the two groups. The in-hospital mortality was $3.3 \%$ (two deaths) in the entire cohort and was not significantly different between the median sternotomy (MS) group and the totally thoracoscopic (TT) group. Most patients in the TT group had their tracheal intubation removed within 24 hours of surgery. The TT group had a diminished requirement for blood transfusion and a reduced 4-day postoperative chest tube drainage amount. The incidence of early major complications, including all-cause death and reoperation due to bleeding, was lower in the TT group. No significant differences were observed in the 7-year survival probability between the two groups.

Conclusions: The encouraging results regarding the perioperative and long-term outcomes of patients who underwent a TT re-MVR show that this approach is particularly beneficial for patients requiring reoperation.

Keywords: Reoperative mitral valve replacement (re-MVR); totally thoracoscopic (TT); minimally invasive cardiac surgery (MICS)

Submitted Apr 20, 2021. Accepted for publication May 28, 2021.

doi: $10.21037 / \mathrm{atm}-21-2407$

View this article at: http://dx.doi.org/10.21037/atm-21-2407 


\section{Introduction}

As patients continue to survive longer after their initial mitral valve surgery (MVS), the need for reoperative mitral valve surgery (re-MVS) is increasing (1). Between 2002 and 2016, the volume of re-MVSs reportedly increased by $10 \%$ each year. And In 2017, over 14\% of patients undergoing MVS had prior MVS (1). However, reoperative cardiac procedures are associated with an increased perioperative risk (2). Traditionally, re-MVS has been performed by median sternotomy (MS), but with the recent advances in surgical techniques, minimally invasive therapy for MVS has become a more popular choice with excellent perioperative and long-term results $(3,4)$. Since the early 1990s, various alternative approaches to MS have been introduced, including partial sternotomy, anterolateral mini-thoracotomy, total thoracoscopy, and transcatheter procedures.

The excellent clinical outcomes of a totally thoracoscopic (TT) initial MVS (5) have rekindled our excitement toward the use of this approach in patients with previous MVS. Although several studies have compared the traditional MS approach with other minimally invasive approaches (6-8), the literature concerning a TT approach for reoperative mitral valve replacement (re-MVR) is limited. Consequently, we sought to evaluate the perioperative and long-term outcomes of patients undergoing a TT re-MVR approach compared with a concurrent cohort who had their procedures completed via resternotomy. We present the following article in accordance with the STROBE reporting checklist (available at http://dx.doi.org/10.21037/atm-212407).

\section{Methods}

\section{Study population}

Between January 2013 and December 2019, 151 consecutive patients underwent re-MVR with or without concomitant tricuspid surgery at the Department of Cardiovascular Surgery of Guangdong Provincial People's Hospital. All patients had previously undergone at least one open MVS via MS. The study population consisted of 48 patients who had their re-MVR performed totally via thoracoscopy while 103 patients had their procedures performed via MS. This retrospective, observational study was conducted according to the Helsinki Declaration (as revised in 2013) and was approved by the ethics committee of Guangdong Provincial People's Hospital (No. KY-Q-2021-088-01). Individual consent for this retrospective analysis was waived.

\section{Surgical procedures}

Our operative technique has been described in detail in a previous publication (5) and is summarized here.

\section{The TT approach}

A main operating port ( $4.0 \mathrm{~cm}$ incision) and a thoracoscopic port $(1.5 \mathrm{~cm}$ incision) were made in the right chest as previously described (5). After systemic heparinization, the cardiopulmonary bypass (CPB) was initiated. Dissection of pleural adhesions was performed if required, and then the pericardium was opened vertically, medial to the phrenic nerve. After the ascending aorta was mobilized and cannulated, a Chitwood clamp was used to clamp the aorta. The surgical field was constantly flushed with carbon dioxide to prevent air embolism. The mitral valve was accessed through a left atriotomy in the interatrial groove, and MVR was then performed under cardioplegia. If we failed to dissect the ascending aorta in cases of dense pericardial adhesion, the procedure was then performed on the beating heart or under ventricular fibrillation (VF). The intracardiac operation was performed via the main operating port using thoracoscopic instruments (Figure 1).

\section{MS}

Standard MVR was performed routinely via resternotomy.

\section{Concomitant procedures}

Concomitant tricuspid valvuloplasty (TVP) was performed as necessary.

\section{Follow-up}

The first follow-up visit was 3 months following hospital discharge. Clinic or telephone follow-ups were then conducted annually. The follow-up aimed to record any adverse events, in particular, death, dysfunction of the prosthetic valve, the need for reoperation, and stroke. The follow-up ended on December 30th, 2020.

\section{Statistical analysis}

Since patients were not randomly assigned to the two approaches for re-MVR, propensity score matching was applied. Propensity scores for each patient were calculated using a logistic regression analysis with 21 covariables. 

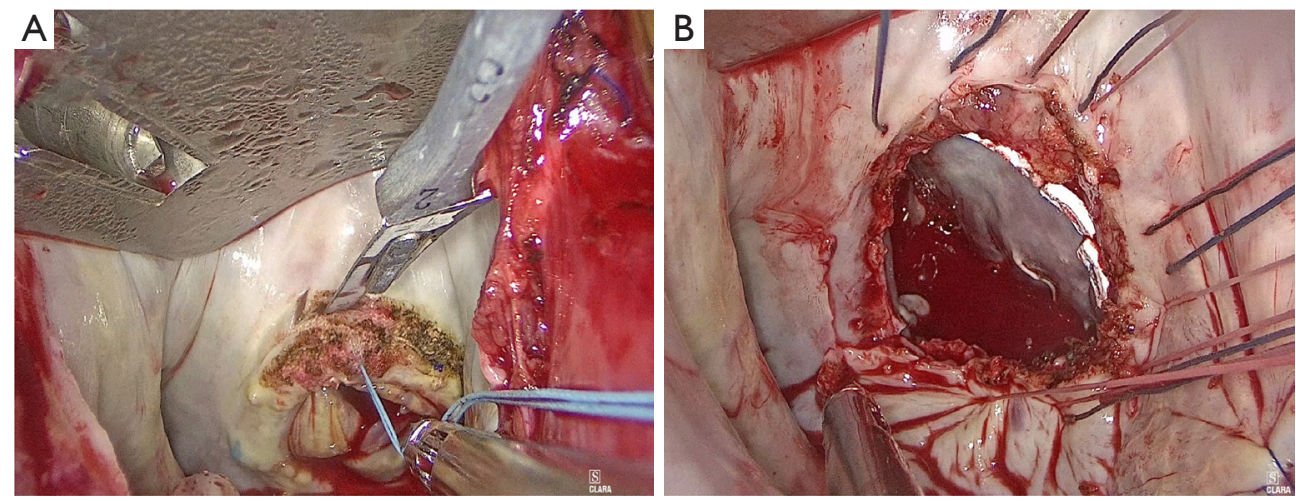

Figure 1 Re-MVR in a patient with a failed bioprosthesis. (A) Slicing along the failed bioprosthesis annulus using a knife; (B) pledgeted sutures are placed on the annulus toward the left ventricle. Re-MVR, reoperative mitral valve replacement.

Patients who underwent a TT re-MVR were matched with patients who underwent MS in a 1:1 ratio through the nearest neighbor matching algorithm. To exclude bad matches, we instituted a caliper of 0.2 of the standard deviation (SD) of the logit of the propensity score (9). The matched sample included 60 patients, evenly distributed between the TT group and the MS group.

Categorical variables are expressed as frequencies and percentages. Continuous variables are presented as mean $\pm \mathrm{SD}(\mathrm{M} \pm \mathrm{SD})$. The Student's $t$-test was used to compare continuous variables between the two groups. Associations between categorical variables were evaluated by using the Chi-squared test or Fisher's exact test as appropriate. Survival curves were generated using the Kaplan-Meier method and compared with the log-rank test. A two-sided P value of $\leq 0.05$ was considered significant. Statistical analysis was performed with $\mathrm{R}(\mathrm{R} \times 64$ version 4.0.2, $\mathrm{R}$ Foundation for Statistical Computing, Vienna, Austria). The package "MatchIt" (10) was used for the propensity score matching.

\section{Results}

\section{Propensity score matching}

A total of 151 patients underwent re-MVR (48: TT; 103: MS) over the 7 years. Baseline characteristics, including New York Heart Association (NYHA) classification, left ventricular end-systolic dimension (LVDs), left ventricular end-diastolic dimension (LVDd), European System for Cardiac Operative Risk Evaluation II (EuroSCORE II), type of procedures, and indicators differed significantly between the patients in the two groups (Table 1). Before matching, the cardiac function of patients in the TT group was better than that of patients in the MS group (NYHA III: $44.7 \%$ vs. $20.8 \%$ ). There was no statistical difference between the two groups in terms of the time interval from previous procedures, number of previous MVSs, previous medical history [such as atrial fibrillation (AF), stroke, etc.], and left ventricular systolic function. The EuroSCORE II score of the MS group was significantly higher than that of the TT group. However, the LVDs and LVDd of the TT group were significantly greater than those of the MS group. The predominant indicators for reoperation in the TT group and MS group were the failure of valvuloplasty (68.8\%) and prosthetic valve dysfunction (bioprosthetic valve degeneration $35.9 \%$, mechanical valve thrombosis $26.2 \%)$, respectively. After propensity score matching, 30 pairs of cases were obtained to reduce the confounding factors that are inherent in observational studies. In the matched cohorts, a reduced standardized mean difference and a well-proportioned distribution of propensity scores were observed.

\section{Operative characteristics and early postoperative outcomes of the matched cohort}

Although the CPB time of the TT group was significantly longer than that of the MS group $(137.57 \pm 40.22$ vs. $177.23 \pm 70.93 \mathrm{~min}, \mathrm{P}<0.05)$, there was a trend toward a shorter operation time in the TT group $(270.80 \pm 83.63$ vs. $287.13 \pm 56.25 \mathrm{~min}, \mathrm{P}=0.38$ ). All procedures in the MS group were successfully performed under arrest. In the TT group, the operation was performed under arrest, on the beating heart, and under VF in 7, 17, and 6 patients, respectively. The most common prosthetic valve type and size used in 
Table 1 Baseline patient characteristics before and after propensity score matching

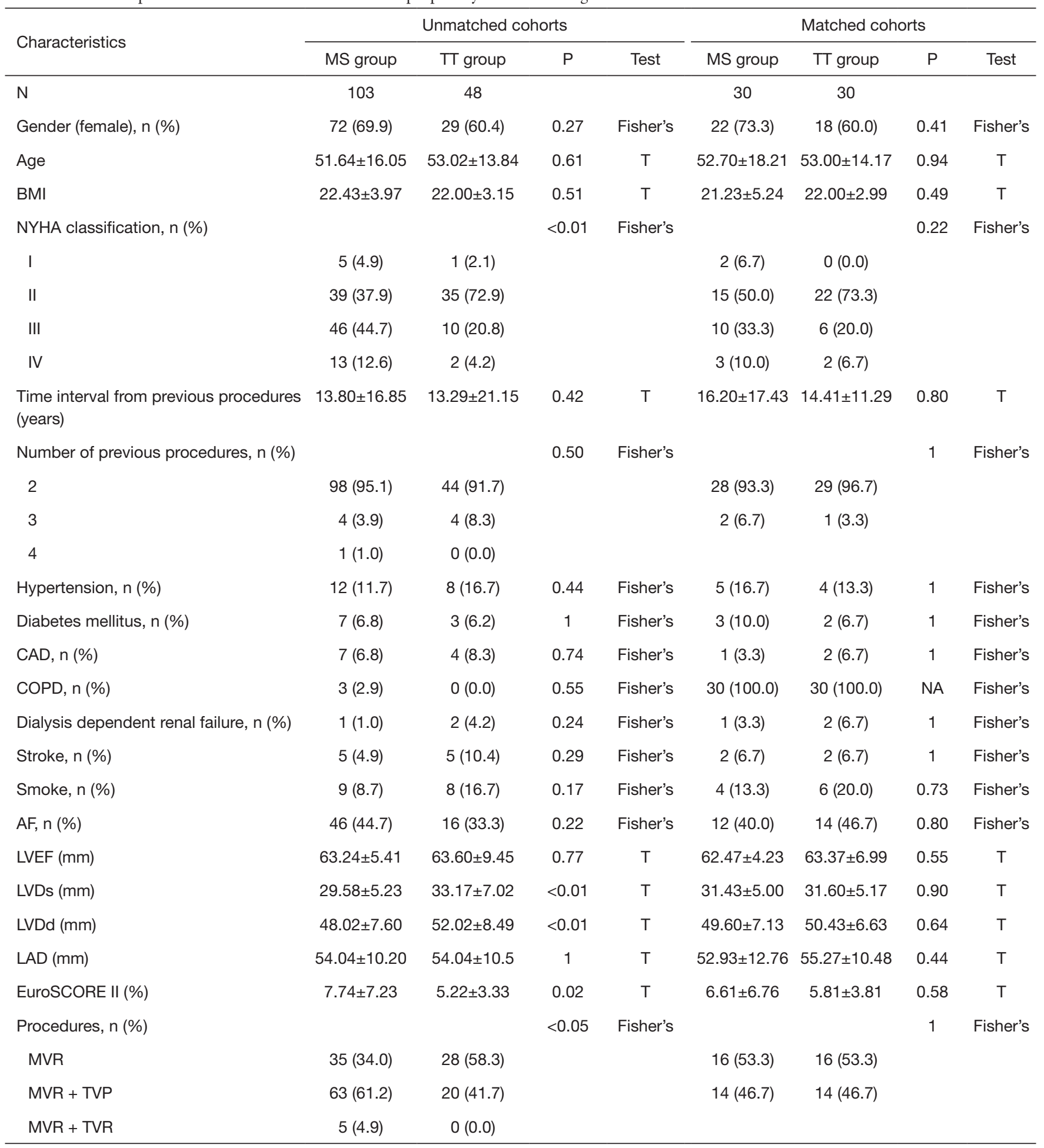

Table 1 (continued) 
Table 1 (continued)

\begin{tabular}{|c|c|c|c|c|c|c|c|c|}
\hline Characteristics & \multicolumn{4}{|c|}{ Unmatched cohorts } & \multicolumn{4}{|c|}{ Matched cohorts } \\
\hline Indications, n (\%) & & & $<0.01$ & Fisher's & & & 0.01 & Fisher's \\
\hline Valve repair failure & $23(22.3)$ & $33(68.8)$ & & & $13(43.3)$ & $18(60.0)$ & & \\
\hline Bioprosthetic valve degeneration & $37(35.9)$ & $8(16.7)$ & & & $13(43.3)$ & $5(16.7)$ & & \\
\hline Paravalvular leakage & $14(13.6)$ & $6(12.5)$ & & & $0(0.0)$ & $6(20.0)$ & & \\
\hline Endocarditis & $2(1.9)$ & $1(2.1)$ & & & $0(0.0)$ & $1(3.3)$ & & \\
\hline
\end{tabular}

MS, median sternotomy; TT, totally thoracoscopic; BMI, body mass index; NYHA, New York Heart Association; CAD, coronary artery disease; COPD, chronic obstructive pulmonary disease; AF, atrial fibrillation; LVEF, left ventricular ejection fraction; LVDd, left ventricular end-diastolic dimension; LVDs, left ventricular end-systolic dimension; LAD, left atrial dimension; EuroSCORE II, European System for Cardiac Operative Risk Evaluation II; MVR, mitral valve replacement; TVP, tricuspid valvuloplasty; TVR, tricuspid valve replacement.

both groups was the mechanical valve, size 27. Although no significant difference was observed, there was a trend toward a shorter ventilation time and a shorter stay in the intensive care unit (ICU) in the TT group. However, most patients in the TT group had their tracheal intubation removed within 24 hours of the operation. The blood transfusion rate of the TT group was lower [7 (23.3\%) vs. 24 (80\%), $\mathrm{P}<0.01$ ], including red blood cells, plasma, and platelets, than the MS group. The chest tube drainage amount within 4 days postoperatively was significantly less in the TT group $(273.97 \pm 223.80$ vs. $684.87 \pm 432.50 \mathrm{~mL}$, $\mathrm{P}<0.01)$. One patient died during hospitalization in each group. Of note, the incidence of severe early complications including reoperation, pneumonia, acute renal failure, and low cardiac output syndrome was significantly lower in the TT group than in the MS group (Table 2).

\section{Comparison of echocardiographic parameters of the matched cohort}

In both groups after propensity score matching, there was no significant difference in LVDs between the preoperative transthoracic echocardiogram (pre TTE) and the postoperative transthoracic echocardiogram (post TTE) within 3 months. However, the LAD and LVDd of the post TTE were significantly reduced compared to that of the pre TTE (Figure 2). This was also further confirmed in the latest transthoracic echocardiogram (latest TTE) during the follow-up period (Table 3). Surprisingly, no significant difference in left ventricular ejection fraction (LVEF) was observed between the pre TTE and latest TTE despite a significant decrease in the LVEF of the post TTE in the MS group. In contrast, comparisons of LVEF between the pre TTE and post TTE, and pre TTE and latest TTE showed no significant differences. It indicated that the TT group may have been superior to the MS group in protecting ventricular systolic function.

\section{Follow-up}

After matching, the follow-up rate and mean follow-

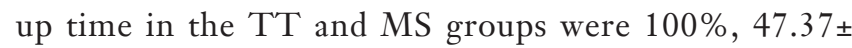

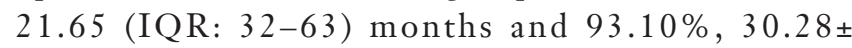
15.25 (IQR: 16-42) months, respectively. Most patients in the MS group underwent their procedures from 2017 to 2019 , so the follow-up time was significantly longer in the TT group. During the follow-up period, the incidence of death, abnormal valve function, and arrhythmia in the two groups were $3.7 \%, 7.41 \%$, and $3.7 \%$ (MS group) and $6.90 \%, 3.45 \%$, and 0 (TT group), respectively.

Before matching, the follow-up rate and mean followup time of the TT and MS groups were $84.10 \%, 45.33 \pm$ 24.66 (IQR: 24-64) months, and $81.37 \%, 39.07 \pm$ 23.28 (IQR: 18-51) months, respectively. The mortality rate and reoperation rate of the MS group were $6.02 \%$ and $1.20 \%$, respectively, while those of the TT group were $10 \%$ and $2.5 \%$, respectively. The incidence of valvular dysfunction, arrhythmia, and stroke was higher in the MS group than in the TT group (Table 4).

To reduce the impact of the difference in the follow-up rate and follow-up time between the two groups, survival analysis was performed and plotted using the Kaplan-Meier 
Table 2 Operative characteristics and early postoperative outcomes of the matched cohort

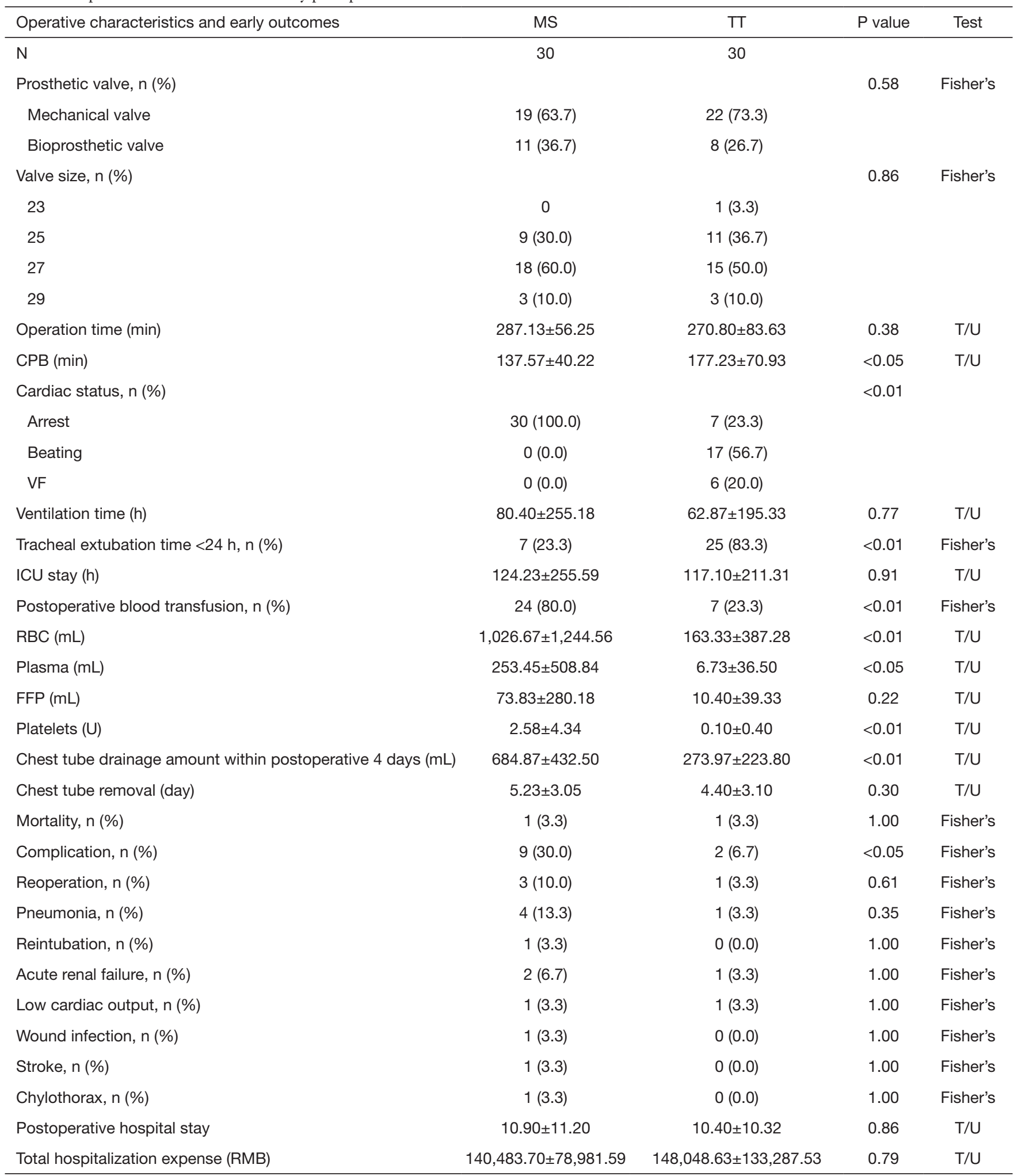

MS, median sternotomy; TT, totally thoracoscopic; CPB, cardiopulmonary bypass; ICU, intensive care unit; VF, ventricular fibrillation; RBC, red blood cell; FFP, fresh frozen plasma. 

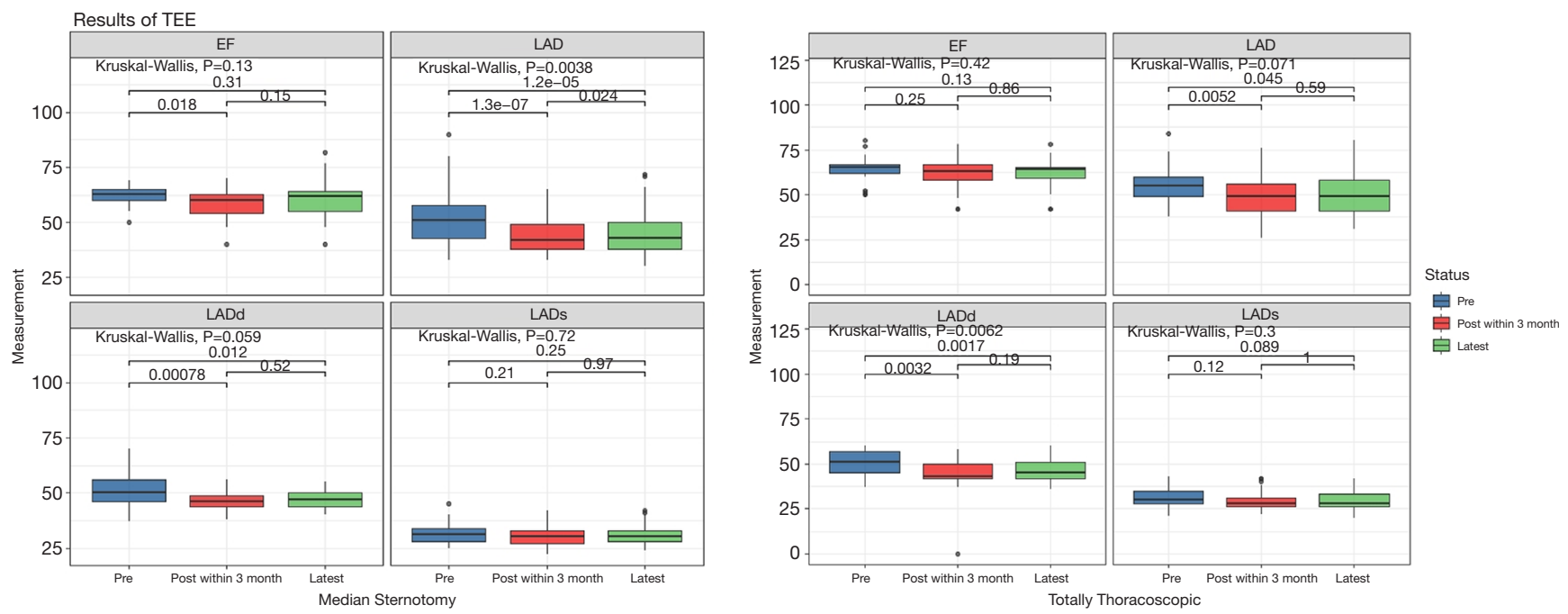

Figure 2 Histograms of the echocardiographic parameters of the matched cohort: pre TTE (blue); post TTE within 3 months (red); latest TTE during the follow-up period (green). Pre TTE, preoperative transthoracic echocardiogram; post TTE, postoperative transthoracic echocardiogram; latest TTE, latest transthoracic echocardiogram.

Table 3 Comparisons of the echocardiographic parameters of the matched cohort

\begin{tabular}{|c|c|c|c|c|c|}
\hline Echocardiography & Preoperative & Postoperative within 3 months & $\mathrm{P}$ value (paired $t$-test) ${ }^{*}$ & Latest & $P$ value (paired $t$-test) \\
\hline \multicolumn{6}{|l|}{ MS } \\
\hline $\mathrm{EF}$ & $62.38 \pm 4.28$ & $58.90 \pm 7.12$ & $<0.05$ & $60.62 \pm 8.98$ & 0.31 \\
\hline LAD & $52.97 \pm 12.99$ & $43.21 \pm 7.57$ & $<0.01$ & $45.86 \pm 10.73$ & $<0.05$ \\
\hline LVDd & $50.00 \pm 6.91$ & $46.59 \pm 4.34$ & $<0.01$ & $47.03 \pm 4.31$ & 0.01 \\
\hline \multicolumn{6}{|l|}{$\pi$} \\
\hline $\mathrm{EF}$ & $64.31 \pm 7.04$ & $62.10 \pm 9.33$ & 0.25 & $61.90 \pm 8.01$ & 0.13 \\
\hline LAD & $55.45 \pm 10.48$ & $49.34 \pm 11.07$ & $<0.01$ & $50.55 \pm 11.32$ & $<0.05$ \\
\hline LVDd & $50.55 \pm 6.76$ & $43.93 \pm 10.04$ & $<0.01$ & $46.41 \pm 5.58$ & $<0.01$ \\
\hline
\end{tabular}

*, preoperative vs. postoperative within 3 months; ", preoperative vs. latest. MS, median sternotomy; TT, totally thoracoscopic; EF, ejection fraction; LAD, left atrial dimension; LVDd, left ventricular end-diastolic dimension; LVDs, left ventricular end-systolic dimension.

method. The difference in survival rate between the two groups was compared by the log-rank method (Figure 3).

Before matching, no significant difference was observed in the 1-year survival [MS group: 96.1\%, 95\% confidence interval (CI): $92.4-100 \%$; TT group: $89.5 \%, 95 \% \mathrm{CI}$ : $81.3-98.7 \%$ ] or 7-year survival (MS group: $81.7 \%$, $95 \%$ CI: $64.1-100 \%$; TT group: $82.9 \%$, 95\% CI: $71.5-96.2 \%)$. After matching, there was also no significant difference in the survival rate between the two groups $(\mathrm{P}=0.18)$.

\section{Survival analysis}

After univariate Cox regression analysis of the individual variables that could affect the postoperative survival rate of patients (Table 5), significant multiple variables were included in the multivariate Cox regression analysis (Figure 4). The analysis revealed that age ( $>65$ years) was an independent risk factor for death. Although the time interval from previous procedures was not an independent 
Table 4 Severe complications occurring during the follow-up period

\begin{tabular}{|c|c|c|c|c|}
\hline Complications & \multicolumn{2}{|c|}{ Matched cohort } & \multicolumn{2}{|c|}{ Unmatched cohort } \\
\hline $\mathrm{N}$ & 27 & 29 & 83 & 40 \\
\hline Death, n (\%) & $1(3.70)$ & $2(6.90)$ & $5(6.02)$ & $4(10.00)$ \\
\hline Reoperation, n (\%) & $0(0.00)$ & $0(0.00)$ & $1(1.20)$ & $1(2.50)$ \\
\hline Arrhythmia, n (\%) & $1(3.70)$ & $0(0.00)$ & $3(3.61)$ & $0(0.00)$ \\
\hline Stroke, n (\%) & $0(0.00)$ & $0(0.00)$ & $1(1.20)$ & $0(0.00)$ \\
\hline
\end{tabular}

MS, median sternotomy; TT, totally thoracoscopic.

A Survival probability of unmatched group Strata $\Psi$ group $=$ Median sternotomy $\Psi$ group $=$ Totally thoracoscopic
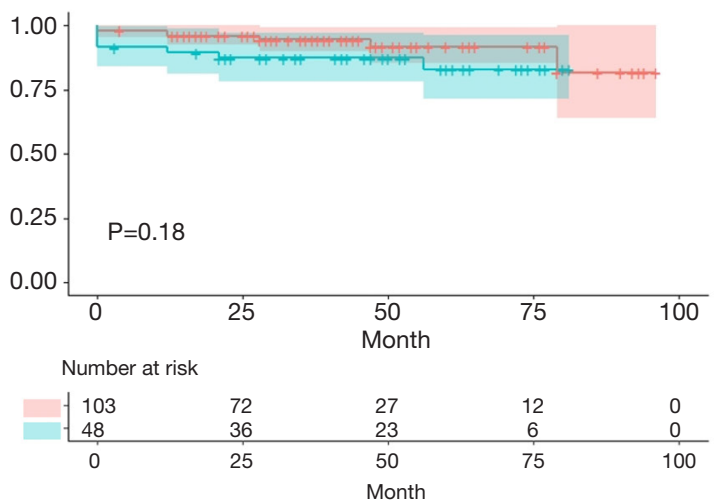

B

Survival probability of matched group

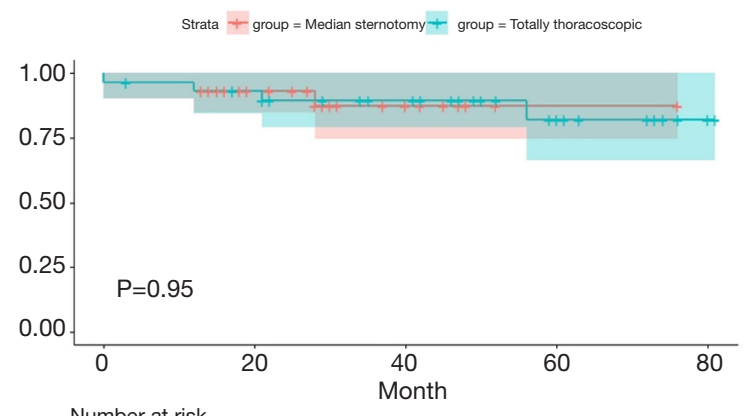

Number at risk

\begin{tabular}{ccccc}
30 & 19 & 9 & 1 & 0 \\
30 & 26 & 19 & 10 & 2 \\
\hline 0 & 20 & 40 & 60 & 80 \\
& & Month & &
\end{tabular}

Figure 3 Kaplan-Meier plot for survival in the propensity score matched (A) and unmatched (B) cohorts.

risk factor for death, the longer the time interval, the higher the hazard ratio (HR) of death. According to the exploratory subgroup analysis, the forest plot showed/no significant difference in the survival rate between the two surgical approaches in each subgroup (Figure 5).

\section{Discussion}

Compared to primary cardiac surgery, re-MVS is associated with a higher risk of mortality (11). In the past, most cardiac surgeons have preferred traditional resternotomy as the surgical approach for re-MVS, as they are more familiar with MS. Since the early 1990s, minimally invasive cardiac surgery (MICS) via various approaches has emerged as an attractive and valid alternative to conventional cardiac surgery using MS. Compared to MS, MICS is associated with a shorter ventilator time, a shorter length of time in
ICU (12), and less need for blood transfusions (13) and, as such, has gained increasing acceptance. In Germany, the percentage of patients undergoing a minimally invasive MVS increased from $13.1 \%$ in 2004 to $45.2 \%$ in 2013 (14). In China, the total number of minimally invasive adult cardiac surgeries in 2019 reached 20,487 cases with an increase of 5,360 cases (35.5\%) compared to 2018 (15). As MICS is now performed in many cardiac centers, several of them have introduced various minimally invasive approaches to re-MVS. In our center, we have a wealth of experience performing primary thoracoscopic MVS, and a TT procedure has become our preferred approach for primary and re-MVS since 2013. This study aimed to evaluate the perioperative and long-term outcomes of patients who had undergone a TT re-MVR.

In the 1980 s, re-MVS had a mortality rate of up to $50 \%$ (16), so the main concerns were severe complications 
Table 5 Univariate and multivariate Cox hazard analysis of risk factors for death

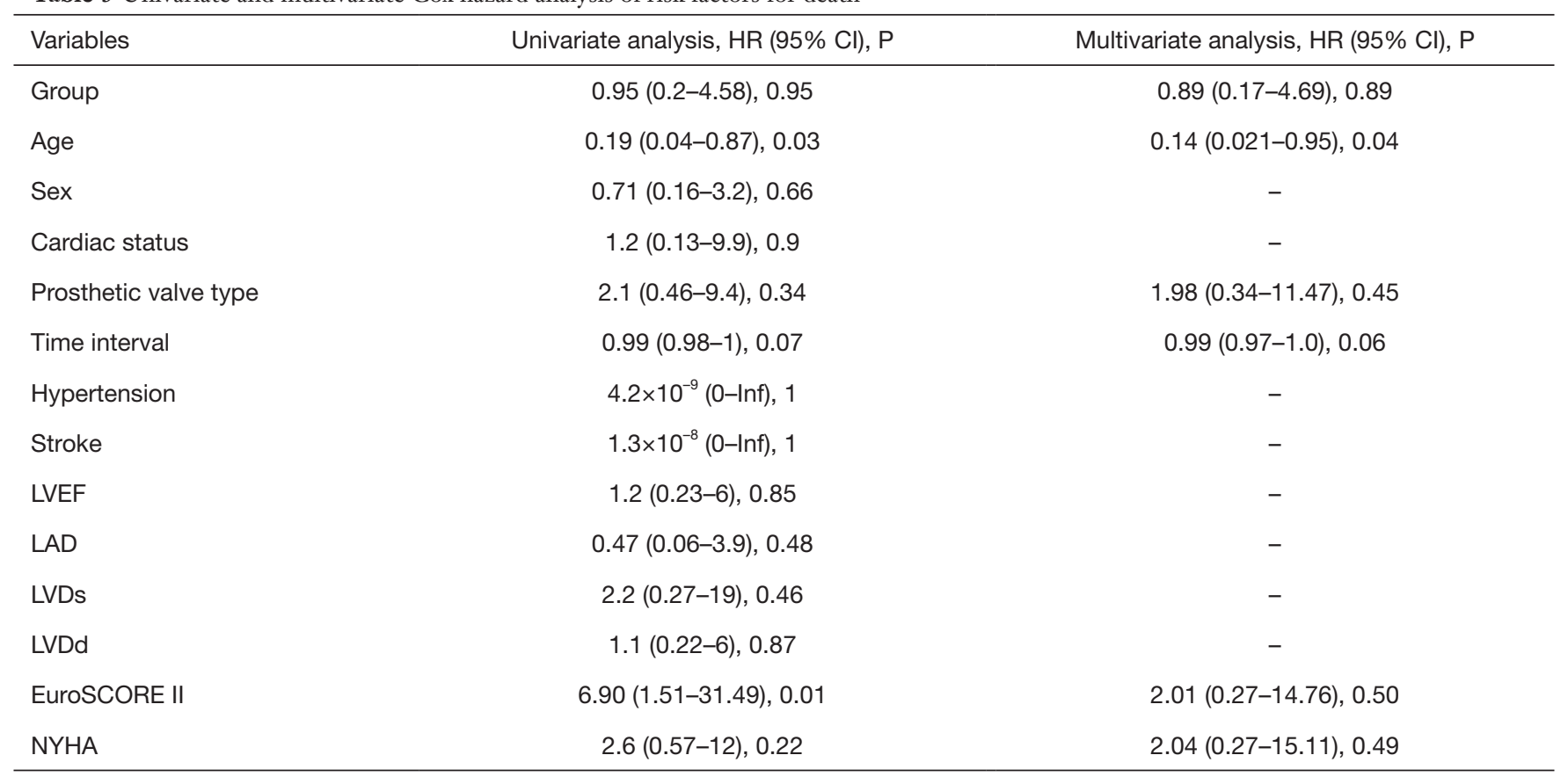

HR, hazard ratio; CI, confidence interval; LVEF, left ventricular ejection fraction; LAD, left atrial dimension; LVDs, left ventricular endsystolic dimension; LVDd, left ventricular end-diastolic dimension; EuroSCORE II, European System for Cardiac Operative Risk Evaluation II; NYHA, New York Heart Association.

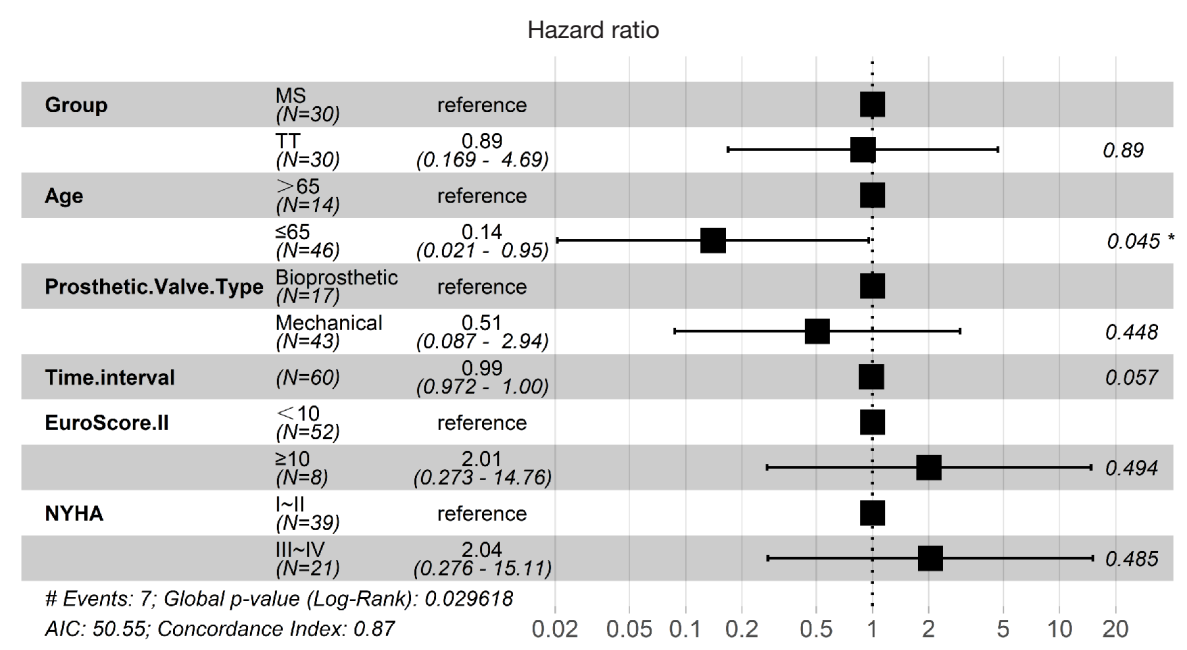

Figure 4 Multivariate Cox hazard analysis plot of the risk factors for death in the matched cohort. EuroSCORE II, European System for Cardiac Operative Risk Evaluation II; NYHA, New York Heart Association.

and mortality. As mentioned in a contemporary review, as the number of re-MVS increased at the rate of $10 \%$ per year from 2002 to 2016, mortality decreased dramatically after an initial rise. And the observed-to-expected ratios, which were calculated using the Society of Thoracic Surgeons risk models for predicted 30-day mortality in re-
MVS, decreased from 1.5 to below 0.75 during the same study period (1). Currently, mortality rates for re-MVS via minimally invasive approaches and resternotomy range from $0 \%$ to $13 \%$ and from $4.2 \%$ to $12 \%$, respectively (17). Several studies have compared the MS approach with right mini-thoracotomy and right infra-axillary thoracotomy 


\begin{tabular}{|c|c|c|c|c|c|}
\hline subgroup & No.of.patients & Median.Sternotomy.n(\%) & Totally.Thoracoscopic.n(\%) & $\mathrm{HR}(95 \% \mathrm{Cl})$ & P for interaction \\
\hline Overall & 60 & $30(30)$ & $30(30)$ & 年 & \\
\hline Age & & & & & 0.67 \\
\hline$<=65$ & 46 & $23(50)$ & $23(50)$ & $1.05(0.07-16.74)$ & \\
\hline$>65$ & 14 & $7(50)$ & $7(50)$ & $1.05(0.07-16.74)$ & \\
\hline Prosthetic.Valve.Type & & & & & 0.3 \\
\hline Bioprosthetic & 17 & $9(52.94)$ & $8(47.05)$ & $0.63(0.06-6.98)$ & \\
\hline Mechanical & 43 & $21(48.83)$ & $22(51.16)$ & $1.79(0.17-18.86)$ & \\
\hline EuroScore.II & & & & & 0.24 \\
\hline$<=10$ & 52 & $26(50)$ & $26(50)$ & $1.81(0.17-19.1)$ & \\
\hline$>10$ & 8 & $4(50)$ & $4(50)$ & $0.4(0.04-4.49)$ & \\
\hline
\end{tabular}

Figure 5 Forest plot depicting the HRs of MS versus TT procedures. MS, median sternotomy; TT, totally thoracoscopic; HR, hazard ratio; CI, confidence interval.

approaches (6-8) for re-MVS, and a meta-analysis shows that a right anterolateral mini-thoracotomy approach was associated with a significantly reduced number of deaths compared to resternotomy (18). So far, however, there have been limited evaluations of a TT approach for re-MVS.

Actually, the TT approach for re-MVR has not been applied widely in clinical. The main factors are the long learning curve, operationally demanding and concerns about safety and effectiveness. In the present study, 151 consecutive patients underwent re-MVR in our institution from January 2013 to December 2019. Among them, 48 patients had their re-MVR performed totally via thoracoscopy while 103 patients had their procedures performed via MS. However, for observational studies, treatment selection is often influenced by subject characteristic. So, it is difficult to rule out bias and confounding. And propensity score matched analysis is a statistical matching technique that estimate the effect of a treatment, policy, or baseline patient characteristic by accounting for the covariates.

After propensity score matching, compared to resternotomy, TT re-MVR was associated with significant lower postoperative blood transfusion rate [7 (23.3\%) vs. 24 $(80 \%), \mathrm{P}<0.01]$. And the chest tube drainage amount within 4 days postoperatively was significantly less in the TT group. And patients underwent TT re-MVR were more likely to wean from the ventilator earlier than those of MS group. There was a trend toward a shorter ventilation time and a shorter stay in the ICU in the TT group. It indicated that TT re-MVR has the advantages of reducing bleeding, avoiding damage to intracardiac structures and prevent potential ventilator-induced lung injury. The incidence of major complication was significantly lower in the TT group. The in-hospital mortality (3.3\%, two deaths) and the 7-year survival in the matched cohort were comparable with that of other centers, and no significant difference was found between the two groups. For the entire cohort, no significant difference was observed in 1 -year survival or 7-year survival between the two groups. This demonstrates that a TT re-MVR approach is feasible and safe. The most interesting aspect of the results is that there was no significant difference in total hospitalization expenses between TT group and MS group $(\mathrm{P}=0.79)$ (See Table 2).

Three technical factors contribute to the safety and feasibility of a TT re-MVR. Firstly, the procedure reduces the possibility of injury to cardiac structures due to the avoidance of resternotomy $(19,20)$. After the right thoracotomy, the mitral valve was well exposed via thoracoscopy. In the TT group, there was a trend toward a shorter operation time despite a longer CPB time. The main reason may be that surgeons took more time to achieve hemostasis in the MS group. The phenomenon of significantly fewer blood transfusions and less chest drainage in the TT group also confirmed this. Secondly, with the advancement in myocardial protection, the procedure can be performed on an empty beating heart or under VF in cases where the aorta cannot be clamped due to heavy adhesion. The additional advantage of this strategy is the elimination of ischemia-reperfusion injury which can arise following aortic cross-clamping and releasing (21). A recent study by Romano and colleagues showed that the beating-heart technique is as safe as the arrested-heart technique, with less blood transfusion and lower ventilator times (22). Hiraoka et al. performed 10 cases of re-MVR under VF by thoracotomy with a three- 
dimensional videoscope in 2013, and their early experience suggested that the procedure was acceptable as an effective alternative (23). Results of our study confirmed this conclusion. Thirdly, the strategy of de-airing is the key to the prevention of perioperative stroke. A propensity score matched study conducted by Ad and colleagues compared the incidence of stroke between minimally invasive MVS under VF with conventional MVS via MS, and no significant difference was observed (24). In our study, to avoid air embolism, we took measures such as continuous carbon dioxide insufflation, insertion of a needle into the ascending aorta to de-air, the intraoperative maintenance of arterial pressure above $50 \mathrm{mmHg}$ to close the aortic valve, and the use of the Trendelenburg position.

With the rapid development of transcatheter valve surgery, transcatheter valve implantation for failed bioprosthetic valves (a valve-in-valve procedure) is emerging as another attractive alternative to conventional surgical valve replacement (25). However, the main causes leading to re-MVR are failed mitral valve repair and structural deterioration of prosthetic valve. And a previous report indicated a high rate of reoperative procedures, including replacement with bioprosthesis $(42.2 \%)$ and replacement with a mechanical valve (39.7\%) (26). For patients who are deemed inappropriate candidates for transcatheter therapy (those with mechanical valve thrombosis, severe paravalvular leakage, infective endocarditis, and young patients with failed mitral valve repair) a TT approach is still an effective, safe, and minimally invasive alternative with the advantages of less trauma, rapid recovery, less need for blood transfusion, and an acceptable long-term outcome.

As this is a retrospective and observational study, we performed propensity score matching analysis to eliminate the inherit selection bias. However, propensity score matching leads to a small study sample size (30 pairs). We acknowledge that it may affect the confidence of the conclusion.

\section{Conclusions}

The encouraging results regarding the perioperative and long-term outcomes of patients who underwent a TT reMVR show that this approach is particularly beneficial for patients requiring reoperation.

\section{Acknowledgments}

Funding: China National Key Research and Development Program(2018YFC1002600); Science and Technology
Program of Guangzhou (202002030039).

\section{Footnote}

Reporting Checklist: The authors have completed the STROBE reporting checklist. Available at http://dx.doi. org/10.21037/atm-21-2407

Data Sharing Statement: Available at http://dx.doi. org/10.21037/atm-21-2407

Conflicts of Interest: All authors have completed the ICMJE uniform disclosure form (available at http://dx.doi. org/10.21037/atm-21-2407). The authors report funding from China National Key Research and Development Program (2018YFC1002600) and Science and Technology Program of Guangzhou (202002030039). The authors have no other conflicts of interest to declare.

Ethical Statement: The authors are accountable for all aspects of the work in ensuring that questions related to the accuracy or integrity of any part of the work are appropriately investigated and resolved. All procedures performed in this study involving human participants were in accordance with the Declaration of Helsinki (as revised in 2013). This study was approved by the Institutional Review Board of Guangdong Provincial People's Hospital (No. KYQ-2021-088-01). Individual consent for this retrospective analysis was waived.

Open Access Statement: This is an Open Access article distributed in accordance with the Creative Commons Attribution-NonCommercial-NoDerivs 4.0 International License (CC BY-NC-ND 4.0), which permits the noncommercial replication and distribution of the article with the strict proviso that no changes or edits are made and the original work is properly cited (including links to both the formal publication through the relevant DOI and the license). See: https://creativecommons.org/licenses/by-nc-nd/4.0/.

\section{References}

1. Mehaffey HJ, Hawkins RB, Schubert S, et al. Contemporary outcomes in reoperative mitral valve surgery. Heart 2018;104:652-6.

2. Cohn LH. Evolution of redo cardiac surgery: review of personal experience. J Card Surg 2004;19:320-4.

3. Grossi EA, Loulmet DF, Schwartz CF, et al. Evolution of 
operative techniques and perfusion strategies for minimally invasive mitral valve repair. J Thorac Cardiovasc Surg 2012;143:S68-70.

4. Grant SW, Hickey GL, Modi P, et al. Propensity-matched analysis of minimally invasive approach versus sternotomy for mitral valve surgery. Heart 2019;105:783-9.

5. Liu J, Chen B, Zhang YY, et al. Mitral valve replacement via minimally invasive totally thoracoscopic surgery versus traditional median sternotomy: a propensity score matched comparative study. Ann Transl Med 2019;7:341.

6. Cao H, Zhou Q, Fan F, et al. Right anterolateral thoracotomy: an attractive alternative to repeat sternotomy for high-risk patients undergoing reoperative mitral and tricuspid valve surgery. J Cardiothorac Surg 2017;12:85.

7. Cao H, Zhou Q, Xue Y, et al. Mitral valve surgery via a right infra-axillary thoracotomy in high-risk reoperative patients. Heart Surg Forum 2020;23:E200-4.

8. Monsefi N, Öztürk M, Shavahatli T, et al. Right minithoracotomy approach in patients undergoing redo mitral valve procedure. Indian J Thorac Cardiovasc Surg 2020;36:591-7.

9. Austin PC. Optimal caliper widths for propensity-score matching when estimating differences in means and differences in proportions in observational studies. Pharm Stat 2011;10:150-61.

10. Ho D, Imai K, King G, et al. Matchit: Nonparametric preprocessing for parametric causal inference. J Stat Softw $20112011 ; 42: 28$.

11. Seeburger J, Borger MA, Falk V, et al. Minimally invasive mitral valve surgery after previous sternotomy: experience in 181 patients. Ann Thorac Surg 2009;87:709-14.

12. Modi P, Hassan A, Chitwood WR Jr. Minimally invasive mitral valve surgery: a systematic review and meta-analysis. Eur J Cardiothorac Surg 2008;34:943-52.

13. Gammie JS, Zhao Y, Peterson ED, et al. J. Maxwell Chamberlain Memorial Paper for adult cardiac surgery. Less-invasive mitral valve operations: trends and outcomes from the Society of Thoracic Surgeons Adult Cardiac Surgery Database. Ann Thorac Surg 2010;90:1401-8, 1410.e1; discussion 1408-10.

14. Lange R, Voss B, Kehl V, et al. Right minithoracotomy versus full sternotomy for mitral valve repair: a propensity matched comparison. Ann Thorac Surg 2017;103:573-9.

15. Wei L, Chen N, Yang Y, et al. The data of Chinese minimally invasive cardiovascular surgery in 2019. Chin J Clin Thorac Cardiov Surg 2021;28:149-53.
16. Husebye DG, Pluth JR, Piehler JM, et al. Reoperation on prosthetic heart valves. An analysis of risk factors in 552 patients. J Thorac Cardiovasc Surg 1983;86:543-52.

17. Fatehi Hassanabad A, Turcotte M, Dennehy C, et al. Contemporary reoperative mitral valve surgery: technical considerations and clinical outcomes. Innovations (Phila) 2020;15:425-39.

18. Daemen JHT, Heuts S, Olsthoorn JR, et al. Right minithoracotomy versus median sternotomy for reoperative mitral valve surgery: a systematic review and meta-analysis of observational studies. Eur J Cardiothorac Surg 2018;54:817-25.

19. Dobell AR, Jain AK. Catastrophic hemorrhage during redo sternotomy. Ann Thorac Surg 1984;37:273-8.

20. Loop FD. Catastrophic hemorrhage during sternal reentry. Ann Thorac Surg 1984;37:271-2.

21. Kitamura T, Stuklis RG, Edwards J. Redo mitral valve operation via right minithoracotomy--"no touch" technique. Int Heart J 2011;52:107-9.

22. Romano MA, Haft JW, Pagani FD, et al. Beating heart surgery via right thoracotomy for reoperative mitral valve surgery: a safe and effective operative alternative. J Thorac Cardiovasc Surg 2012;144:334-9.

23. Hiraoka A, Kuinose M, Totsugawa T, et al. Mitral valve reoperation under ventricular fibrillation through right mini-thoracotomy using three-dimensional videoscope. J Cardiothorac Surg 2013;8:81.

24. Ad N, Holmes SD, Shuman DJ, et al. Minimally invasive mitral valve surgery without aortic cross-clamping and with femoral cannulation is not associated with increased risk of stroke compared with traditional mitral valve surgery: a propensity score-matched analysis. Eur J Cardiothorac Surg 2015;48:868-72; discussion 872.

25. Goode D, Dhaliwal R, Mohammadi H. Transcatheter mitral valve replacement: state of the art. Cardiovasc Eng Technol 2020;11:229-53.

26. Kwedar K, McNeely C, Zajarias A, et al. Outcomes of early mitral valve reoperation in the medicare population. Ann Thorac Surg 2017;104:1516-21.

Cite this article as: Wei P, Liu J, Ma J, Zhang Y, Chen Z, Liu Y, Tan T, Wu H, Chen J, Zhuang J, Guo H. Long-term outcomes of a totally thoracoscopic approach for reoperative mitral valve replacement: a propensity score matched analysis. Ann Trans Med 2021;9(12):987. doi: 10.21037/atm-21-2407 